\title{
II danno da emotrasfusione: dieci sentenze delle Sezioni Unite della Cassazione. II parte
}

\author{
Marianna Pulice ${ }^{1}$ \\ 1 Avvocato, Foro di Cosenza
}

\begin{abstract}
The first part of this article, published in the 2008; $4(2)$ issue of this Journal, examined ten judgements of the United Sections of the Court of Cassation concerning negligent transfusion of infected blood, with transmission of hepatitis virus, B or C, or HIV. In this second part, the Author analyses some of these legal decisions with attention to prescription of the judgement, worsening of the infected patient, value of Commissions proceedings, and causal connection.
\end{abstract}

Keywords: transfusion of infected blood, negligence, legal decisions

Injury from blood transfusion: ten decisions of the United Sections of the Court of Cassation. II part Pratica Medica \& Aspetti Legali 2009; 3(2): 69-73

\section{INTRODUZIONE}

Facendo seguito al lavoro "Il danno da emotrasfusione: dieci sentenze delle Sezioni Unite della Cassazione" pubblicato sul numero di novembre 2008 di questa stessa testata [1], riprendiamo in questo articolo la disamina delle questioni giuridiche affrontate dalle dieci sentenze della Suprema Corte di Cassazione e orbitanti attorno al tema del danno da emotrasfusione o da emoderivati (le sentenze a cui si fa riferimento sono riportate in Tabella I).

In particolare si cercherà ora di analizzare alcuni temi specifici: la prescrizione delle azioni volte a ottenere il risarcimento, il caso del peggioramento del paziente infettato e la valenza del verbale redatto dalla Commissioni all'uopo preposte.

Infine si cercherà, alla luce delle autorevoli pronunce in esame, di scomporre il tema del nesso causale per capire come e quando è imputabile una responsabilità al medico o alla struttura sanitaria, pubblica o privata, presso cui avviene il trattamento sanitario.

\section{DESCRIZIONE DEI CASI}

\section{CASO 1}

Tizia, in quanto affetta sin dalla nascita da talassemia major, viene sottoposta a varie trasfusioni e solo dopo molti anni scopre di essere affetta da epatite C. Presenta pertanto istanza al Ministero della Salute per l'indennizzo di cui alla legge n. 210/1992, che le viene accordato dopo 6 anni. Presenta altresì al Ministero la domanda di risarcimento di tutti i danni subiti in conseguenza delle trasfusioni di sangue infetto; il Tribunale accoglie la domanda e la Corte d'Appello rigetta l'impugnazione del Ministero che decide di proporre ricorso in Cassazione.

\section{CASO 2}

Caio viene ricoverato d'urgenza presso l'ospedale dove gli viene diagnosticata una gastrite e dove viene sottoposto a trasfusioni di sangue. Il giorno successivo, dimesso, si fa ricoverare presso una clinica privata, dove subisce un intervento di rese- 


\begin{tabular}{|ll|}
\hline Corte di Cassazione, SS.UU., sentenza n. 576/2008 & Infezione da HIV in paziente emofiliaco \\
\hline Corte di Cassazione, SS.UU., sentenza n. 577/2008 & $\begin{array}{l}\text { Infezione da HCV in seguito a trasfusioni in } \\
\text { occasione di un intervento chirurgico }\end{array}$ \\
\hline Corte di Cassazione, SS.UU., sentenza n. 578/2008 & Infezione da HIV \\
\hline Corte di Cassazione, SS.UU., sentenza n. 579/2008 & Infezione da HCV in paziente talassemico \\
\hline Corte di Cassazione, SS.UU., sentenza n. 580/2008 & Infezione da HCV \\
\hline Corte di Cassazione, SS.UU., sentenza n. 581/2008 & Infezione da HBV, HCV e HIV in diversi pazienti \\
\hline Corte di Cassazione, SS.UU., sentenza n. 582/2008 & Infezione da HCV in paziente sottoposto a trasfusioni \\
\hline Corte di Cassazione, SS.UU., sentenza n. 583/2008 & Infezione da HCV in paziente talassemico \\
\hline Corte di Cassazione, SS.UU., sentenza n. 584/2008 & Infezione da HIV in seguito a emotrasfusione \\
\hline Corte di Cassazione, SS.UU., sentenza n. 585/2008 & Infezione da HCV in paziente talassemico \\
\hline
\end{tabular}

Tabella I. Sentenze della Corte di Cassazione relative a infezioni insorte in seguito alla somministrazione di sangue infetto

zione gastrica, a seguito del quale si rendono necessarie altre trasfusioni. Il paziente accerta successivamente di essere affetto da epatite $\mathrm{C}$ e adisce quindi le vie legali al fine di chiedere la condanna del soggetto che, tra la struttura pubblica o privata, risulti responsabile del contagio da epatite $\mathrm{C}$ a seguito di trasfusione con sangue infetto.

\section{LEGGE 210/92. INDENNIZZO A FAVORE DEI SOGGETTI DANNEGGIATI DA COMPLICANZE DI TIPO IRREVERSIBILE A CAUSA DI VACCINAZIONI OBBLIGATORIE E TRASFUSIONI}

\section{Articolo 1}

1. Chiunque abbia riportato, a causa di vaccinazioni obbligatorie per legge o per ordinanza di una autorità sanitaria italiana, lesioni o infermità, dalle quali sia derivata una menomazione permanente della integrità psicofisica, ha diritto a un indennizzo da parte dello Stato, alle condizioni e nei modi stabiliti dalla presente legge

2. L'indennizzo di cui al comma 1 spetta anche ai soggetti che risultino contagiati da infezioni da HIV a seguito di somministrazione di sangue e suoi derivati, nonché agli operatori sanitari che, in occasione e durante il servizio, abbiano riportato danni permanenti alla integrità psicofisica conseguenti a infezione contratta a seguito di contatto con sangue e suoi derivati provenienti da soggetti affetti da infezione da HIV

3. I benefici di cui alla presente legge spettano altresì a coloro che presentino danni irreversibili da epatiti posttrasfusionali

4. I benefici di cui alla presente legge spettano alle persone non vaccinate che abbiano riportato, a seguito $e$ in conseguenza di contatto con persona vaccinata, i danni di cui al comma 1; alle persone che, per motivi di lavoro o per incarico del loro ufficio o per potere accedere ad uno Stato estero, si siano sottoposte a vaccinazioni che, pur non essendo obbligatorie, risultino necessarie; ai soggetti a rischio operanti nelle strutture sanitarie ospedaliere che si siano sottoposti a vaccinazioni anche non obbligatorie

\section{QUESTIONI GIURIDICHE AFFRONTATE}

\section{CASO 1}

\section{La prescrizione dei danni lungo latenti}

Nelle ipotesi di danno da emotrasfusione o da emoderivati non è facile determinare il dies a quo, ossia il giorno della decorrenza del termine di prescrizione delle azioni volte a ottenere il risarcimento dei danni. Lordinamento giuridico, infatti, nel caso del mancato esercizio di un diritto (in questo caso, il diritto di agire in giudizio) per un certo periodo di tempo (predeterminato dalla legge) prevede l'estinzione del diritto stesso; questo è l'istituto della prescrizione. La legge ha stabilito che la prescrizione comincia a decorrere dal giorno in cui il diritto può essere fatto valere; benché tale disciplina sia inderogabile, essa va comunque interpretata in relazione alle diverse fattispecie concrete degli specifici casi. I danni da emotrasfusione o emoderivati, ad esempio, rappresentano un caso molto particolare e complesso in quanto si tratta di cosiddetti "danni lungo latenti", ovverosia danni che possono manifestarsi dopo un lungo lasso di tempo.

Nel 1942 il legislatore, nel Codice civile, ha affidato la questione dell'exordium praescriptionis a indicazioni scarne e alquanto generiche: l'art. 2935 cc, con l'espressione "la prescrizione comincia a decorrere dal giorno in cui il diritto può essere fatto valere», si presta a molteplici interpretazioni e l'art. 2947, comma 1, stabilisce solo che «il diritto al risarcimento del danno derivante da fatto illecito si prescrive in cinque anni dal gionno in cui il fatto si è verificato». Dunque, diversamente dal Codice francese (Code Napoléon, a cui il nostro Co- 
dice si è ispirato) e dal Codice del 1865, per l'istituto in esame si è scelto di prescindere da qualsiasi ragione oggettiva o soggettiva del ritardo della vittima nell'instaurazione delle pretese, di non tener conto delle difficoltà nella disponibilità della prova per l'attore in conseguenza del decorso del tempo e di far coincidere il dies a quo con la verificazione dell'evento dannoso. Ma da tutto questo si evince un quadro sicuramente sbilanciato a favore del convenuto, quadro che è stato sin da subito avversato dagli interpreti, in particolare proprio nel campo del danno alla persona dove, negli anni '70, vi è stato l'ingresso dirompente, ad opera della giurisprudenza, del danno biologico, e lo sgretolamento dell'art. $2059 \mathrm{cc}$, con conseguente ribaltamento di alcuni schemi legislativi.

Proprio in tema di prescrizione dei danni lungo latenti si iniziò ad affermare [2] che occorreva spostare il dies a quo dalla verificazione del fatto all'esteriorizzazione del danno, passando quindi da un limite rigido e penalizzante a un limite "mobile", e si iniziò così una rilettura dell'art. 2947 cc mediante un'opera di affinamento del concetto di “conoscibilità del danno" [3,4].

In sintesi, l'evoluzione giurisprudenziale ha portato a interpretare l'espressione legislativa "verificarsi del danno", contenuta nell'art. 2947 cc, nel senso che il danno deve essere "oggettivamente percepibile e riconoscibile». Non è dunque sufficiente che il paziente abbia la mera consapevolezza di stare male; egli deve, al contrario, poter dimostrare la gravità delle conseguenze lesive del danno sulla sua salute anche dal punto di vista della rilevanza giuridica.

Tuttavia, in tempi successivi, anche queste considerazioni sono state ritenute fuorvianti, poiché la conoscenza del danno non necessariamente comporta la conoscibilità del fatto giuridicamente rilevante ai fini di un'azione risarcitoria; si è pertanto detto che l'art. $2947 \mathrm{cc}$ va interpretato nel senso che il dies a quo deve decorrere dal momento in cui la malattia è percepita o può essere percepita come danno ingiusto conseguente a un comportamento doloso o colposo di un terzo, usando l'ordinaria diligenza e tenuto conto delle conoscenze scientifiche. Si è quindi è detto che vanno applicati il principio della conoscibilità del danno e il principio della rapportabilità causale [5-7].

Le Sezioni Unite della Corte di Cassazione, con le sentenze 576/2008, 579/2008, 580/2008 e $584 / 2008$, hanno aderito a questa impostazione giurisprudenziale ma con un'opportuna avvertenza: non è un'apertura alla rilevanza della mera conoscibilità soggettiva del danneggiato.

Infatti, la conoscibilità viene ancorata a due parametri oggettivi quali l'ordinaria diligenza e il livello di conoscibilità scientifica in relazione all'uomo medio. Sono quindi stati introdotti un parametro interno e uno esterno, entrambi verificabili dal giudice senza scendere in un'indagine di tipo psi- cologico con tutte le conseguenze che ne sarebbero derivate.

\section{I peggioramenti}

Una volta fissati i principi in tema di prescrizione di origine giurisprudenziale, occorre però tenere presente una scrupolosa avvertenza che la Suprema Corte ha voluto fare per l'ipotesi in cui il soggetto, a seguito di vaccinazioni, trasfusioni o somministrazioni di emoderivati, abbia contratto una malattia in costante evoluzione peggiorativa. Nel giudizio relativo a questa ipotesi si cerca spesso di far valere la teoria del cosiddetto "termine mobile della prescrizione".

A tal proposito la Corte di Cassazione distingue due situazioni:

- un fatto illecito, che, dopo un primo evento lesivo, determina ulteriori conseguenze pregiudizievoli;

- un fatto illecito permanente in cui il comportamento lesivo non si esaurisce uno actu, ma perdura nel tempo oltre la produzione del danno e lo alimenta per tutta la sua durata.

Per il primo caso la Cassazione civile a sezioni unite, richiamando un costante indirizzo giurisprudenziale [8-11], ha affermato che la prescrizione dell'azione risarcitoria per il danno inerente alle ulteriori conseguenze dannose decorre dal verificarsi di queste ultime solo a condizione che le medesime non costituiscano un mero sviluppo e un aggravamento del danno già insorto, ma la manifestazione di lesione nuova e autonoma rispetto a quella esteriorizzatasi con l'esaurimento dell'azione del responsabile, dunque il semplice peggioramento di una lesione in atto non può spostare il termine iniziale di prescrizione.

\section{La valenza dei verbali delle commissioni}

Presso gli ospedali militari la legge ha previsto l'istituzione di commissioni medico-ospedaliere composte da ufficiali medici cui viene affidato il compito di stabilire l'esistenza del nesso causale tra vaccinazioni, trasfusioni o somministrazioni di emoderivati e danni alla salute della persona, ai fini dell'indennizzo a favore dei soggetti danneggiati da complicanze di tipo irreversibile.

Le suddette commissioni redigono dei verbali contenenti dei giudizi diagnostici sulla cui valenza molto si è discusso nel tempo. La Cassazione civile, sulla base di un indirizzo già presente nelle pronunce dei giudici di legittimi [12-15], ha operato una distinzione fondamentale: i fatti che la commissione attesta essere avvenuti in sua presenza, o essere stati dalla stessa compiuti, fanno piena prova, ex art. 2700 cc, mentre le valutazioni, 
le diagnosi o comunque le manifestazioni di scienza o di opinione in essi contenute costituiscono materiale indiziario soggetto al libero apprezzamento del giudice, il quale può valutarne l'importanza ai fini della prova, ma non può mai attribuire loro il valore di accertamento vero e proprio.

Dunque, al di fuori del procedimento amministrativo per la concessione dell'indennizzo di cui alla legge, tali verbali hanno lo stesso valore di qualunque altro documento redatto da un pubblico ufficiale fuori dal giudizio civile e in questo prodotto.

Un altro problema che da sempre si è posto è quello relativo alla valenza di tale responso in relazione alla prescrizione della sentenza; in particolare ci si è chiesto se la comunicazione del verbale, in cui viene formulato il giudizio diagnostico, possa essere considerato il dies a quo della prescrizione della relativa azione giudiziaria.

La suprema Corte di Cassazione, nella sentenza $579 / 2008$, ha previsto che, in linea generale, non può ritenersi che solo con la comunicazione di tale responso inizi a decorrere la prescrizione. Tenuto conto che l'indennizzo è dovuto solo in presenza di danni irreversibili da vaccinazioni, emotrasfusioni o somministrazioni di emoderivati, appare ragionevole ipotizzare che, dal momento della proposizione della domanda amministrativa, la vittima del contagio deve comunque aver avuto una sufficiente percezione della malattia e delle possibili conseguenze dannose, percezione la cui esattezza viene confermata solo con la certificazione emessa dalle commissioni mediche.

Il supremo organo giustifica tale impostazione sottolineando che una diversa impostazione offre effettivamente il destro al creditore per dilatare a suo piacere il corso della prescrizione, dato che potrebbe portare ad affermare che il dies a quo inizi anche a decorrere a causa già iniziata, negando l'effetto interrutivo connaturato alla proposizione dell'azione e che si rischia di enfatizzare il ruolo della consulenza medico-legale (effettuata peraltro in riferimento al diverso procedimento di liquidazione dell'indennizzo). Inoltre, è illogico ritenere che il decorso del termine di prescrizione possa iniziare dopo che la parte si è comunque attivata per chiedere un indennizzo per lo stesso fatto lesivo, pur nella diversità tra diritto all'indennizzo e diritto al pieno risarcimento di tutte le conseguenze del fatto dannoso.

\section{CASO 2}

\section{II nesso causale}

Un tema critico su cui dottrina e giurisprudenza civile e penale si sono sempre confrontate e scontrate è quello della causalità giuridica. Le sentenze n. 576-585 del 2008 hanno dato una certa accele- razione a questo tema; in particolare hanno ribadito delle tappe ermeneutiche fondamentali che si sono raggiunte nel tempo.

In una causa per responsabilità professionale medica, accertare l'esistenza del nesso condizionalistico tra la condotta del medico e l'evento del danno è il fulcro della vicenda giudiziaria attorno al quale ruotano tutte le altre questioni giuridiche. Come opportunamente sottolineato dai giudici ermellini, la causalità in campo civile e quella nel campo penale presentano caratteri morfologici e funzionali diversi.

Le Sezioni Unite della Corte di Cassazione, dopo un lungo lavorio giurisprudenziale, sono approdate a una posizione unanime: la sentenza a sezioni unite n. 30328/2002 (cosiddetta sentenza Franzese) [16] ha stabilito che non è consentito dedurre automaticamente dal coefficiente di probabilità espresso dalla legge statistica la conferma, o meno, dell'ipotesi accusatoria sull'esistenza del nesso causale, poiché il giudice deve verificarne la validità nel caso concreto, sulla base delle circostanze del fatto e dell'evidenza disponibile, così che, all'esito del ragionamento probatorio che abbia altresì escluso l'interferenza di fattori alternativi, risulti giustificata e processualmente certa la conclusione che la condotta omissiva del medico è stata condizione necessaria dell'evento lesivo con «alto o elevato grado di credibilità razionale» o "probabilità logica». Dunque, si mantiene il ricorso al giudizio controfattuale per l'accertamento del nesso causale e il grado di probabilità richiesto è prossimo alla certezza, da accertare sulla base di tutte le circostanze del caso concreto.

Le sezioni penali auspicarono subito una conformità di tale posizione anche da parte delle sezioni civili, le quali, invece, basandosi proprio sulle diversità strutturali delle due forme di responsabilità, non hanno aderito.

Ma la sentenza con cui le sezioni civili hanno voluto fornire agli operatori del diritto un contro altare alla sentenza Franzese è stata la n. 21619 del 16 ottobre 2007, in cui si è stabilito che il concetto di causalità in sede civile non coincide con quello applicato dal giudice penale, atteso che i caratteri morfologici e funzionali del nesso eziologico si strutturano secondo modelli di analisi ampiamente diversificati in seno a ciascuno dei due sottosistemi di responsabilità. La causalità civile ordinaria si attesta, difatti, sul versante della probabilità relativa (o "variabile"), caratterizzata dall'accedere a una soglia probabilistica meno elevata rispetto a quella penale, secondo modalità (anche) semantiche che, specie in sede di perizia medico-legale, possono assumere molteplici forme espressive, senza che questo debba, peraltro, vincolare il giudice a una formula peritale e senza che egli perda la sua funzione di operare una selezione di scelte del "giuridicamente rilevante" in un determina- 
to momento storico e con riferimento al singolo, specifico (per certi versi "unico") caso concreto, che ben può prescindere, a fronte dell'altrettanto specifica evidenza probatoria, dalle leggi e dalle risultanze di tipo statistico. La causalità civile, dunque, obbedisce alla logica del "più probabile che non".

Sono proprio le teorie della regolarità causale e del novus actus interveniens, affiancate dalla teoria dello scopo della norma violata, che le Sezioni Unite, nel gennaio 2008, hanno ribadito e applicato ai casi concreti sottoposti al supremo consesso riconfermandone così la piena attualità.

Alla luce di ciò, dunque, il nesso di causalità non è accertato come nel campo penale secondo il criterio dell'elevato grado di credibilità razionale che è prossimo alla "certezza", ma consiste nella relazione probabilistica concreta tra comportamento ed evento dannoso, secondo il criterio (ispirato alla regola della normalità causale) del "più probabile che non"; esso si distingue dall'indagine diretta all'individuazione delle singole conseguenze dannose (finalizzata a delimitare, a valle, i confini della già accertata responsabilità risarcitoria) e prescinde da ogni valutazione di prevedibilità o previsione da parte dell'autore, valutazione che va compiuta soltanto in una fase successiva ai fini dell'accertamento dell'elemento soggettivo, ovvero della colpevolezza.

Nella fattispecie della sentenza 21619/2007 la Suprema Corte ha confermato la sentenza di merito che aveva ravvisato il nesso causale tra il comportamento omissivo del Sanitario che aveva ritardato di inviare il paziente presso un centro di medicina iperbarica e l'aggravamento delle lesioni subite dal paziente che probabilmente avrebbe potuto essere evitato. Il nesso causale rappresenta, in sede civile, la misura della relazione probabilistica concreta, svincolata da ogni riferimento soggettivo, tra comportamento e fatto dannoso; esso consente di individuare i termini dell'astratta riconducibilità delle conseguenze dannose delle proprie azioni in capo all'agente, secondo un principio guida formulato in termini di rispondenza, da parte dell'autore del fatto illecito, delle conseguenze che "normalmente" discendono dal suo atto, a meno che non sia intervenuto un nuovo fatto rispetto al quale egli non ha il dovere o la possibilità di agire (in tal senso si parla di teoria della regolarità causale e del novus actus interveniens).

\section{BIBLIOGRAFIA}

1. Pulice M. Il danno da emotrasfusione: dieci sentenze delle Sezioni Unite della Cassazione. Pratica Medica \& Aspetti Legali 2008; 2: $185-9$

2. Cassazione civile, sentenza n. 1716 del 24 marzo 1979

3. Cassazione civile, sentenza n. 8845 del 12 agosto 1995

4. Cassazione civile, sentenza n. 5913 del 9 maggio 2000

5. Cassazione civile, sentenza n. 2645 del 21 febbraio 2003

6. Cassazione civile, sentenza n. 12287 del 5 luglio 2004

7. Cassazione civile, sentenza n. 10493 del 8 maggio 2006

8. Cassazione civile, sentenza n. 21500 del 7 novembre 2005

9. Cassazione civile, sentenza n. 6515 del 2 aprile 2004

10. Cassazione civile, sentenza n. 17940 del 25 novembre 2003

11. Cassazione civile, sentenza n. 7937 del 10 giugno 2000

12. Cassazione civile, sentenza n. 13449 del 20 luglio 2004

13. Cassazione civile, sentenza n. 10128 del 25 giugno 2003

14. Cassazione civile, sentenza n. 10128 del 25 giugno 2003

15. Cassazione civile, sentenza n. 7201 del 12 maggio 2003

16. Corte di Cassazione, SS.UU., sentenza n. 30328 del 10 luglio 2002

\section{CORRESPONDING AUTHOR}

Avv. Marianna Pulice, e-mail:avv.pulice@alice.it 